\title{
Predictive factors of hamstring tendon regeneration and functional recovery after harvesting: a prospective follow-up study
}

Mathijs AM Suijkerbuijk, MD, Max Reijman, Ph.D. ${ }^{1}$, Edwin HG Oei, MD, Ph.D. ${ }^{2}$, Belle L van Meer, MD, Ph.D. ${ }^{1,4}$, Ewoud RA van Arkel, MD, Ph.D. ${ }^{3}$, Duncan E Meuffels, MD, Ph.D. ${ }^{1}$

${ }^{1}$ Department of Orthopaedic Surgery, Erasmus MC, University Medical Center Rotterdam, the Netherlands

${ }^{2}$ Department of Radiology \& Nuclear Medicine, Erasmus MC, University Medical Center Rotterdam, the Netherlands

${ }^{3}$ Department of Orthopaedic Surgery, Haaglanden Medical Center, The Hague, the Netherlands ${ }^{4}$ Department of Sports Medicine, Haaglanden Medical Center, The Hague, the Netherlands 


\section{ABSTRACT}

Background - Semitendinosus and gracilis tendons may regenerate after harvesting for ligament reconstruction procedures. However, predictive factors of tendon regeneration and the extent of functional recovery remain unclear.

Purpose - To identify predictive factors for hamstring tendon regeneration and examine the morbidity of non-regenerated hamstring tendons.

Study design - Cohort study

Methods - 79 of the 154 patients who were included in a prospective follow-up study, underwent reconstruction of the anterior cruciate ligament entailing the hamstring tendons and met the following inclusion criteria: (1) ACL rupture diagnosed by physical examination and MRI; (2) MRI within 6 months after trauma; (3) age between 18 and 45 years; and (4) two-years follow-up MRI data available. Hamstring tendon regeneration was assessed as complete if a tendon-like structure could be visualized at level of joint line or more cranially. Patient characteristics such as age, gender, body mass index, alcohol or nicotine use, activity-level (Tegner scores) and functional instability (one-leg hoptest) at baseline and two years follow-up were evaluated to determine predictive factors for tendon regeneration or to examine functional recovery of hamstring tendon regeneration.

Results - At two years follow-up $67.1 \%$ of the patients showed regeneration of semitendinosus tendons, $81.0 \%$ of gracilis tendons and $59.5 \%$ of both tendons. Aging (Odds Ratio (OR) 0.92 change per year of age, 95\% confidence interval (CI) 0.84 to $0.99, p=0.03$ ) and smoking (OR $0.20,95 \% \mathrm{CI} 0.05$ to $0.77, \mathrm{p}=0.02$ ) significantly decreased the likelihood of semitendinosus tendon regeneration. No predictive factor was found for gracilis tendon regeneration. Regeneration of both the semitendinosus and gracilis tendon was negatively related with smoking (OR 0.22, 95\% CI 0.06 to $0.79, p=0.02$ ). Compared to pre-operative results, patients without regeneration showed post-operatively similar VAS scores during physical activity, similar Tegner scores and a significant decrease of the upper leg circumference. Regardless the regeneration status, one-leg hop-test results significantly increased at two years follow-up.

Conclusions - Hamstring tendon regeneration occurs less frequently in older patients and in smokers. However, absence of regenerated tendons does not seem to cause a loss-of-function.

Clinical relevance - Pre-operative assessment of regeneration chances and an indication of patient's importance of hamstring tendon regeneration.

Key Terms - hamstring tendon regeneration, predictive factors, functional outcome, recovery

What is known about the subject - Hamstring tendon regeneration has been known for a long time and it has been investigated extensively. However, currently

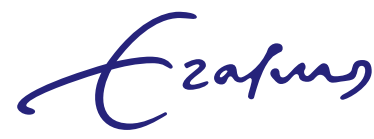


it is unknown what factors affect regeneration chances and, because of conflicting evidence, the importance for patients such as morbidity remains unclear.

What this study adds to existing knowledge - This is the first study that investigated predictive factors for hamstring tendon regeneration and that was able to identify significantly correlated factors. Furthermore, this study assessed functional recovery in patients with and without hamstring tendon regeneration based on both patient reported outcome measurements, and one-leg hoptest and thigh circumferences measurements.

\section{INTRODUCTION}

Anterior cruciate ligament (ACL) rupture is a common sports-related injury of the knee. Estimations of annual incidences reach up to approximately 5 to 8 per 10,000 persons. ${ }^{26,31}$ Numerous graft choices exist for ACL reconstruction, such as hamstring tendons autografts and bone-patellar tendon-bone (BPTB) autografts. Due to donor-site morbidity and patellar tendon ruptures with the use of BPTB autografts, hamstring tendon autografts are a commonly employed option. ${ }^{1,15,22,43}$

Cross et al. were the first describing the potential of hamstring tendons to regenerate after harvesting procedures for ACL reconstructions. ${ }^{12}$ In a previous study, it is described that after having harvested the hamstring tendons, semitendinosus and gracilis tendons regenerate in at least $70 \%$ of the patients. ${ }^{37}$ Currently, it is unknown why some tendons lack the capacity to regenerate. ${ }^{37}$ Mechanical load and controlled mobilization are related with a beneficial effect on tendon recovery after injury. 4, 42, 45 On the contrary, smoking ${ }^{23}$, aging ${ }^{29,} 33$ and the use of alcohol ${ }^{16}$ are related with tendon healing failure. The role of non-steroid anti-inflammatory drugs (NSAIDs) in healing processes remains unclear. ${ }^{14,} 32$ However, the available literature does not describe predictive factors for hamstring tendon regeneration specifically, which could be considered as a different process compared with tendon healing.

A systematic review previously reported about the morbidity and function loss of non-regenerated hamstrings. ${ }^{37}$ Currently, the exact mechanism of the absence of hamstring tendon regeneration is unclear. Several cases have been described in which patients experienced a persistent sharp pain in the dorsal aspect of the thigh in the early stage after surgery. This may be caused by the rupturing of the regenerated structure. ${ }^{28}$ Another explanation might be that the human body suspends its own regenerating efforts in case of non-functional tissue, resulting in a removal of the newly formed, but dysfunctional tissue. Although different studies investigated the clinical response to hamstring tendon regeneration, its consequences remain unclear because of conflicting evidence. A systematic review previously summarized studies that examined the effect of tendon regeneration

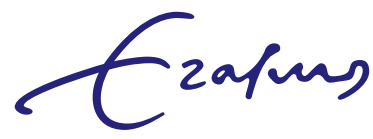


on hamstring strength and function. ${ }^{38}$ This study reported conflicting evidence regarding the relationship between regeneration status and deep knee flexion. Some studies reported a deep knee flexion deficit in patients without regeneration ${ }^{11,} 30$, whereas other studies contradict this finding. ${ }^{13,19,27}$ In addition, there is no consensus about the clinical relevance of the number of regenerated hamstring tendons. Some studies suggest that the extent of deep knee flexion deficits is limited if both tendons regenerate. ${ }^{11}$ Other studies did not find a relationship between the number of regenerated tendons and strength deficits. ${ }^{19}$

Nevertheless, insight in determinants of hamstring tendon regeneration and its clinical consequences is relevant for several reasons. First of all, patients voice concerns about harvesting tendons of functional muscles and possible accompanying functional deficits. If predicting factors are identified, the chances of hamstring tendon regeneration could be estimated more accurately. This may affect the choice of hamstring tendons as an autograft, as well as insight in the clinical consequences of regenerated hamstring tendons. After all, knowledge of determinants for hamstring tendon regeneration may lead to life style modification prior to surgery and changes in rehabilitation programs after surgery.

The aim of the current study was to (1) identify predictive factors for hamstring tendon regeneration and (2) examine the effect of tendon regeneration on hamstring strength and function.

\section{METHODS}

\section{Study Population}

Between January 2009 and November 2010 patients were included in the X study: a prospective multicenter cohort study with 2 years of follow-up. Patients were recruited from 3 hospitals in [country]: [hospital A], [hospital B]; and [hospital C] Delft. Inclusion criteria for [study X] were (1) ACL rupture diagnosed by physical examination and MRI; (2) MRI made within 6 months after trauma; and (3) age between 18 and 45 years old. Patients who did not speak Dutch, those with previous ACL injury or intra-articular knee trauma or surgery, those with disabling co-morbidity and those with already osteoarthritic changes on X-ray (Kellgren and Lawrence grade $>0$ ) at baseline were excluded. Patients were treated operatively or non-operatively independent of the study, according to the decision of the treating physician according to the Dutch ACL guideline. ${ }^{24}$ In the current study, operatively treated patients were included when two-years follow-up MR images, completed questionnaires and data of physical examination of all patients at baseline and two-years follow-up were available. Patients were excluded if the initial treatment was other than an ACL reconstruction entailing the hamstring tendons. Written

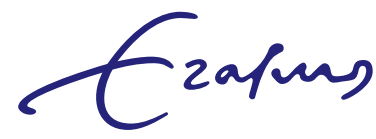


informed consent was obtained from all included patients and the institutions' Medical Ethics Committee approved the study.

\section{Measurements}

Two-years follow-up MRI scans were acquired on a 1.5 Tesla MRI scanner. The patient's legs were positioned in a neutral position using a dedicated knee coil. Details of MR imaging parameters are shown in Table 1.

Table 1. Parameters of MR imaging

\begin{tabular}{lll}
\hline MRI Pulse Sequence & Slice thickness & TR/TE \\
\hline Sagittal and coronal proton density TSE sequence & $3 \mathrm{~mm}$ & $2700 / 27 \mathrm{~ms}$ \\
Coronal T2-weighted TSE sequence with fat saturation & $3 \mathrm{~mm}$ & $5030 / 71 \mathrm{~ms}$ \\
Axial proton density and T2-weighted TSE sequence & $3 \mathrm{~mm}$ & $3500 / 25 / 74 \mathrm{~ms}$ \\
Sagittal 3D water excitation double-echo steady state & $1.5 \mathrm{~mm}$ & $21.35 / 7.97 \mathrm{~ms}$ \\
\hline
\end{tabular}

TSE (Turbo Spin Echo)

Hamstring tendon regeneration was evaluated by an intensively trained researcher (..), who was blinded for clinical information. Hamstring tendon regeneration was assessed at two years follow-up in patients that underwent surgical ACL reconstruction using the hamstring tendons. Equivocal cases were discussed with a musculoskeletal radiologist (..) and a sports medicine trained orthopaedic surgeon (..), both with more than ten years of experienc, and solved with consensus. Hamstring tendon regeneration was assessed at two years follow-up in patients that underwent surgical ACL reconstruction using the hamstring tendons. If regenerated tendons could be visualized at the level of joint line or more cranially, regeneration was assessed as complete. If no neotendons could be visualized on any MR image on any level, this was considered as no regeneration. Therefore, 4 different subgroups of regeneration can be distinguished: 1 . regeneration of both the semitendinosus and gracilis tendon, 2. regeneration of only the semitendinosus, or 3. gracilis tendon, 4. no regeneration of both tendons.

\section{Harvesting procedure}

After an oblique skin incision just medial to the tibial tuberosity, the subcutaneous tissue was dissected to expose the sartorius fascia. A reversed L-shaped incision on this fascia was made to free the whole pes anserinus. The gracilis and semitendinosus tendons were divided from the conjoined tendon of the pes anserinus and whip stitched. Both tendons were harvested using a closed tendon stripper. The sartorius fascia was then sutured in its anatomical position. No drains were used.

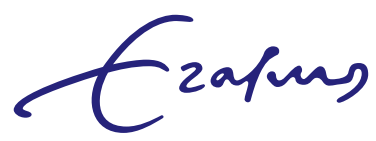




\section{Rehabilitation}

Rehabilitation consisted of full weight bearing and protective crutches use for 6 weeks. No immobilisation or brace was applied. Return to play was considered appropriate in concurrence with the advice of the physiotherapist on average at 8-9 months post-surgery. No specific functional or quantitive protocol as f.i. isokinetic testing was obligatory.

\section{Data collection}

All included patients were requested to complete several questionnaires. One trained medical doctor (BM) who was blinded for the regeneration status, performed standardized physical examination and history taking at baseline and two years follow-up. To evaluate determinants for hamstring tendon regeneration and the clinical consequence of non-regenerated tendons, the following factors and outcome measurements were documented:

- Patient characteristics: gender, age and BMI at baseline. The role of the patient's gender in the process of tendon re-growth remains unclear. ${ }^{37}$ Aging seems to affect tendon regeneration negatively. ${ }^{29}, 33$ No data about the correlation of BMI and tendon regeneration was available. Therefore, patient's BMI was determined and subdivided into three different groups: $\mathrm{BMI}<25 \mathrm{kgm}^{-2}, 25 \mathrm{kgm}^{-2}$ $<\mathrm{BMI}<30 \mathrm{kgm}^{-2}$ and $\mathrm{BMI}>30 \mathrm{kgm}^{-2}$

- Mechanical load: mechanical load is associated with a beneficial effect on hamstring tendon regeneration. 4, 42, 45 Therefore, pre-injury Tegner scores and Tegner scores at two-years follow-up were analyzed as a derivative of mechanical load.

- Hospital: some studies suggest an effect of surgical proceedings and therefore the surgeon may be a potential factor that affects regeneration capacity. ${ }^{37}$

- Intoxications: smoking ${ }^{23}$ and the use of alcohol ${ }^{16}$ seem to negatively affect regeneration changes. The effect of NSAIDs on regeneration remains unclear. ${ }^{14,} 32$

- Vascular status: diabetes mellitus (DM) complicates wound healing and has negative effects on tendon healing processes in animal studies. ${ }^{3,}{ }^{10}$ Moreover, adequate blood supply has shown to be an important factor for ligament healing. 5

- Clinical consequences

o All patients completed the following questionnaires regarding pain, sports activity and knee function: Visual Analogue Score for knee pain (VAS) both at rest and at physical activity ${ }^{20}$, Tegner score (pre-trauma level) ${ }^{20}$, Lysholm $^{6,7,21}$ and International Knee Documentation Committee (IKDC) scores $^{18,41,34}$

o One-leg hoptest (OLHT) was performed and the upper leg circumference of the affected knee was determined

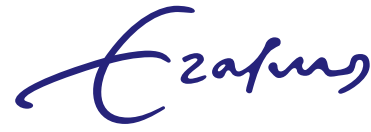




\section{Statistical analysis}

All statistical analyses were performed with IBM SPSS Statistics for Windows (version 21.0, IBM Corp., Armonk, NY). Descriptive statistics were used to describe baseline characteristics. Selection of variables was based on the available literature. To analyze predictive factors for hamstring tendon regeneration, the study population was subdivided into three groups based on the regeneration status. Multivariable binomial logistic regressions were used to calculate odds ratios (OR) and 95\% confidence intervals $(95 \% \mathrm{CI}$ ) for determinants of regeneration of hamstring tendon. Qualitative variables were coded in the following way: gender (man 0, woman 1), smoking (no 0, yes 1), alcohol use (no 0, yes 1), NSAID use (no 0, yes 1). Positive predictive values were calculated for the determinants that had a significant relationship in the multivariable model for hamstring tendon regeneration. Factors were tested for multicollinearity. To determine clinical recovery in case of hamstring tendon regeneration outcomes of three questionnaires and physical examination in four different regeneration subgroups (1. regeneration of both the semitendinosus and gracilis tendon, 2. regeneration of only the semitendinosus, or 3. gracilis tendon, 4. no regeneration of both tendons) and the conservative treated group (control) were compared. Patients that were treated non-operatively were used as controls to examine clinical performance of native tendons after a ruptured ACL. Differences between baseline and two-years follow-up scores were statistically tested using paired t-tests. Significance was tested for $p$-value $<0.05$. To determine the interobserver variability 20 randomly chosen scans were re-assessed by a blinded second observer (..), and an intraclass correlation coefficient (ICC; two-way random effects model, absolute agreement) was calculated

\section{RESULTS}

\section{Study population}

Of the 143 of whom MR imaging at two-years follow-up was available, the baseline characteristics are presented in Table 2.

During the two years follow-up period, 93 patients underwent an ACL reconstruction procedure. A surgical procedure entailing hamstring-tendon grafts was performed in 87 patients (93.5\%), BPTB in 4 patients $(4.3 \%)$ and a combination of hamstring-tendon and allograft in 2 patients (2.2\%). Of 79 patients that underwent an ACL reconstruction using the hamstring tendons post-operative MR imaging was available after a two years follow-up period. At two years follow-up semitendinosus tendon and gracilis tendon regenerated in 53 (67.1\%) and 64 (81.0\%) patients, respectively. No tendon regeneration was reported in 9 (11.4\%) patients.

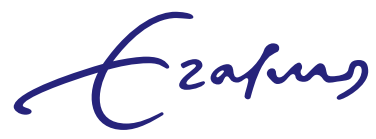


An overview of the regeneration subgroups is displayed in Figure 1. A schematic overview of the flow chart of inclusion of eligible patients is given in Figure 2

Table 2. Patient characteristics at baseline

\begin{tabular}{ll}
\hline Characteristic & $\mathrm{n}=143$ \\
\hline Age (years) & $25.2(21.4-32.6)$ \\
Gender (male) - $\mathrm{n}(\%)$ & $94(65.7)$ \\
$\mathrm{BMI}\left(\mathrm{kg} / \mathrm{m}^{2}\right)$ & $23.9(22.0-26.2)$ \\
Injured side (right) - $\mathrm{n}(\%)$ & $76(53.1)$ \\
Pre-trauma Tegner score & $9(7-9)$ \\
Upper leg circumference of index knee $(\mathrm{cm})$ & $46.7(43.0-48.0)$ \\
One leg hop test of index leg $(\mathrm{cm})$ & $55.0(25.0-85.0)$ \\
\hline
\end{tabular}

Data are presented as median (interquartile range), unless otherwise indicated BMI (body mass index), cm (centimeters)

A

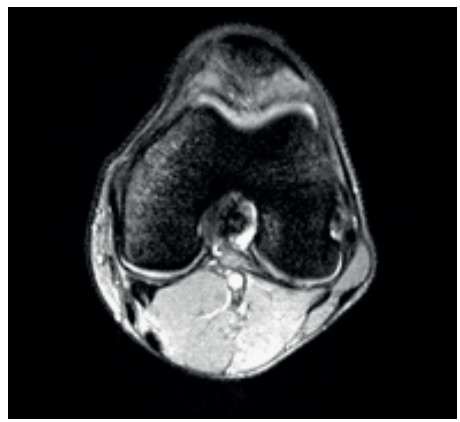

C

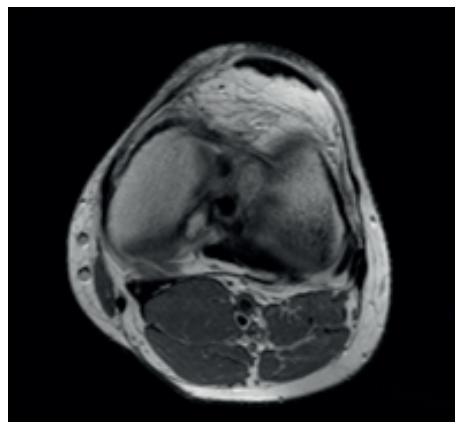

B

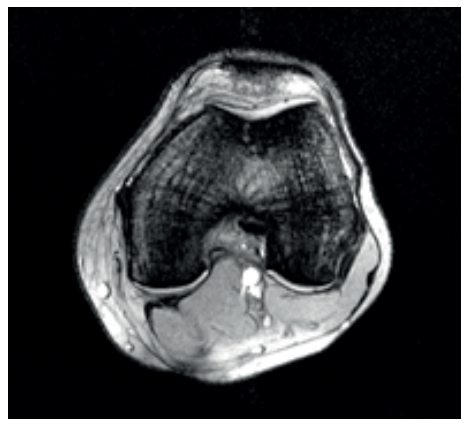

$\mathrm{D}$

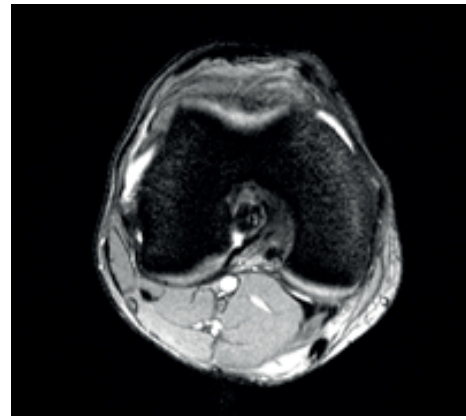

ST; Semitendinosus, G; Gracilis

Figure 1. Representative MR images at joint line level after hamstrings harvesting

A Left knee with regeneration of the semitendinosus and gracilis tendon

B Left knee without regeneration of the semitendinosus and gracilis tendon

C Left knee with only regeneration of the gracilis tendon

D Right knee with only regeneration of the semitendinosus tendon 


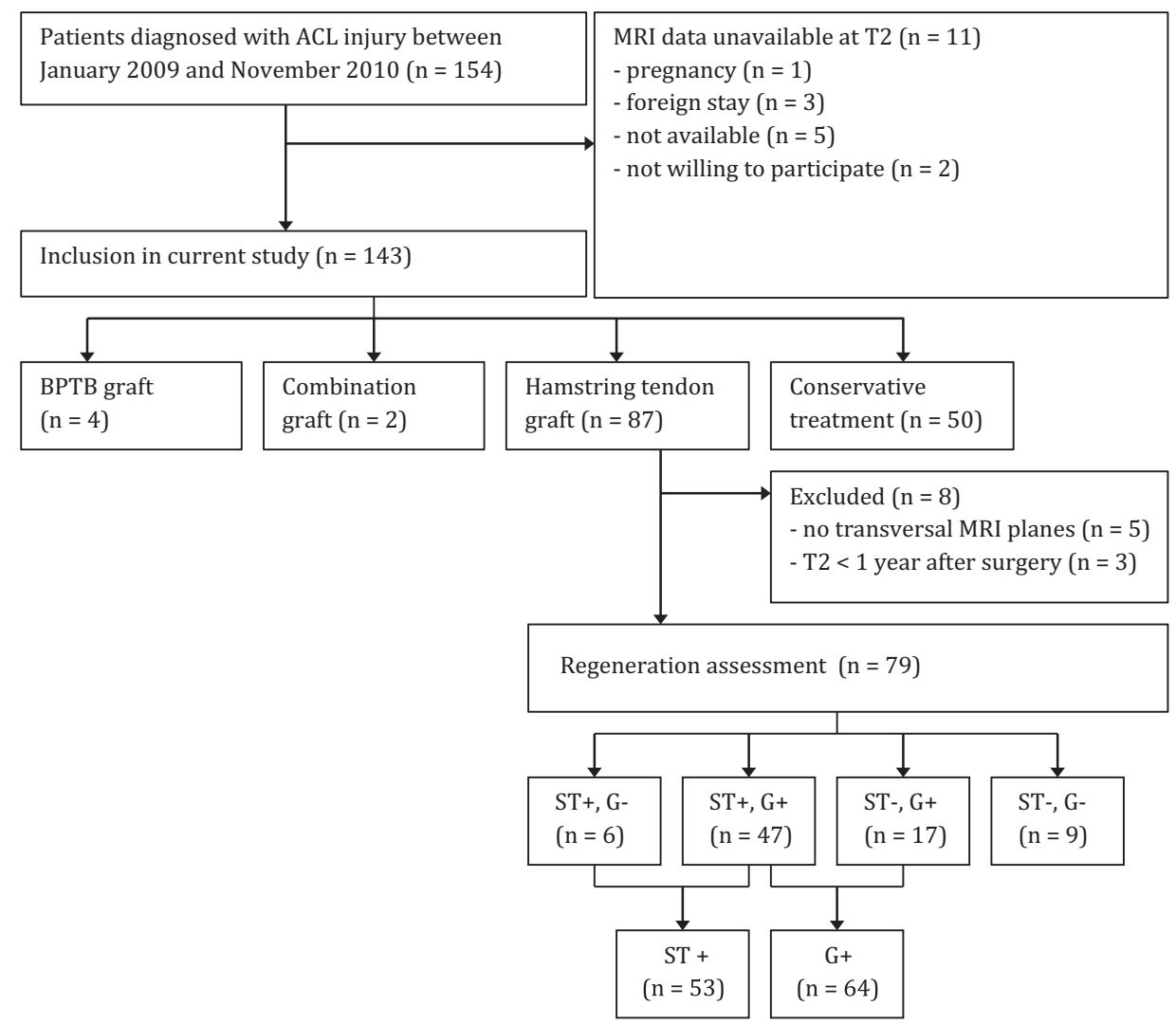

Figure 2. Flowchart

ST (semitendinosus), G (gracilis), + indicates regeneration, - indicates no regeneration

\section{Predictive factors}

Predictive factors in case of regeneration of the semitendinosus tendon $(n=53)$, gracilis tendon $(n=64)$ and both the semitendinosus and gracilis were examined $(n=47)$. Regeneration of the semitendinosus tendon was significantly related with age (OR 0.92 per change per year, $95 \% \mathrm{CI} 0.84$ to $0.99, \mathrm{p}=0.03$ ) and smoking status (OR 0.20, 95\% CI 0.05 to 0.77, p= 0.02). Gracilis tendon regeneration was not related with any of the analyzed predictive factors. Regeneration of both tendons was negatively related with patient's smoking status (OR $0.22,95 \% \mathrm{CI} 0.06$ to $0.79, \mathrm{p}=0.02$ ). An overview of the correlations is presented in Table 3. Because only two patients were suffering from diabetes mellitus and no patients were known with an abnormal cardiovascular status, we did not analyze those determinants for hamstring tendon regeneration outcome. Coefficients of determination varied from $26 \%$ for semitendinosus tendons to $31 \%$ for semitendinosus and gracilis tendons. No multicollinearity was detected. 
Table 3. Multivariable analysis of possible predictive factors for hamstring tendon regeneration $(n=79)$

\begin{tabular}{|c|c|c|c|c|c|c|}
\hline & \multicolumn{2}{|l|}{$S T+(n=53)$} & \multicolumn{2}{|l|}{$G+(n=64)$} & \multicolumn{2}{|l|}{$S T+/ G+(n=47)$} \\
\hline & OR $(95 \% \mathrm{CI})$ & p-value & OR $(95 \% \mathrm{CI})$ & p-value & OR $(95 \% \mathrm{Cl})$ & $\mathrm{p}$-value \\
\hline Gender & $1.7(.54-5.6)$ & .35 & $1.4(.38-5.4)$ & .60 & $.69(.23-2.0)$ & .50 \\
\hline Age & $.92(.84-.99)$ & $.03^{*}$ & $1.0(.92-1.1)$ & .92 & $.95(.88-1.0)$ & .16 \\
\hline NSAID use & $.57(.15-2.2)$ & .42 & $1.1(.23-5.1)$ & .93 & $.52(.15-1.9)$ & .31 \\
\hline \multicolumn{7}{|l|}{ BMI } \\
\hline \multicolumn{7}{|l|}{$-<25 \mathrm{kgm}^{-2}$} \\
\hline$-25-30 \mathrm{kgm}^{-2}$ & $1.6(.43-6.3)$ & .47 & $2.1(.46-9.3)$ & .35 & $1.8(.52-6.1)$ & .36 \\
\hline$->30 \mathrm{kgm}^{-2}$ & $.27(.02-3.8)$ & .33 & $1.0(.06-17)$ & .99 & $.27(.02-3.6)$ & .32 \\
\hline Smoking & $.20(.05-.77)$ & $.02^{*}$ & $.40(.10-1.6)$ & .19 & $.22(.06-.79)$ & $.02^{*}$ \\
\hline Alcohol & $1.3(.36-4.9)$ & .67 & $.86(.21-3.5)$ & .83 & $1.7(.50-5.7)$ & .41 \\
\hline \multicolumn{7}{|l|}{ Surgeon } \\
\hline \multicolumn{7}{|l|}{ - Surgeon 1} \\
\hline - Surgeon 2 & $1.1(.17-7.1)$ & .91 & $3.0(.31-30)$ & .34 & $2.4(.41-14)$ & .33 \\
\hline - Surgeon 3 & $.67(.08-5.4)$ & .71 & $2.6(.21-33)$ & .45 & $1.6(.22-11)$ & .65 \\
\hline - Surgeon 4 & $.21(.01-6.2)$ & .37 & N/A & & $.60(.02-15)$ & .75 \\
\hline - Surgeon 5 & $.54(.11-2.6)$ & .44 & $1.3(.24-6.7)$ & .79 & $1.3(.31-5.5)$ & .71 \\
\hline - Surgeon 6 & $1.7(.20-15)$ & .62 & $\mathrm{~N} / \mathrm{A}$ & & $3.5(.45-27)$ & .23 \\
\hline
\end{tabular}

ST, semitendinosus; G, gracilis; +, indicates regeneration; -, indicates no regeneration; N/A, not available

${ }^{*}$ p-value $<.05$

For the significant determinants after multivariable analyses, the positive predictive values for tendon regeneration are shown in Table 4.

Table 4 Positive predictive values of tendon regeneration

\begin{tabular}{|c|c|c|c|c|c|c|c|}
\hline & & \multicolumn{3}{|c|}{$\mathrm{ST}+$} & \multicolumn{3}{|c|}{$\mathrm{ST}+/ \mathrm{G}+$} \\
\hline & & \multicolumn{3}{|c|}{ Prior chance: $67.1 \%(53 / 79)$} & \multicolumn{3}{|c|}{ Prior chance: $59.5 \%$ (47/79) } \\
\hline & & Yes & No & PPV & Yes & No & PPV \\
\hline \multirow[t]{2}{*}{ Smoking } & Yes & 9 & 11 & .45 & 7 & 13 & .35 \\
\hline & No & 44 & 15 & & 40 & 19 & \\
\hline
\end{tabular}

Based on the multivariate binomial logisitic regression analysis, a prediction rule for regeneration of the semitendinosus and both tendons was designed. The change of semitendinosus tendon regeneration can be predicted using the following formula:

$$
P(\text { regeneration semitendinosus })=\frac{e^{2.245-(0.094 \times a g e)+1.4(\text { smoking })}}{1+e^{2.245-(0.094 \times a g e)+1.4(\text { smoking })}}
$$


The chance of regeneration of both tendons can be estimated using

$$
P(\text { regeneration semitendinosus } \wedge \text { gracilis })=\frac{e^{-.619+1.363(\text { smoking })}}{1+e^{-.619+1.363(\text { smoking })}}
$$

In case a patients smokes, the number 1 should be filled in the formula. Whereas if the patients does smoke, the number 0 should be filled in.

\section{Clinical consequences}

To analyze clinical consequences of tendon regeneration, the study population was subdivided into patients with complete regeneration of the semitendinosus tendon $(n=53)$, complete regeneration of the gracilis tendon $(n=64)$, complete regeneration of both tendons $(n=47)$, no regeneration of both tendons $(n=9)$ and patients that were treated non-operatively $(n=50)$. VAS scores at physical activity significantly decreased in all groups at two years follow-up (all p-values <.001), except for the patients that showed no regeneration of both tendons $(p=.14)$. Before trauma, Tegner scores were significantly higher in all groups compared to Tegner scores at two years follow up, except from the patients with no regeneration of both tendons. Furthermore, the circumference of the upper leg decreased significantly from 47.1 $\mathrm{cm}$ to $45.5 \mathrm{~cm}$ (difference $1.6,95 \% \mathrm{CI}$ of difference $0.46-2.8, \mathrm{p}=.01$ ) in patients with no regeneration of the semitendinosus and gracilis, whereas patients with regeneration of at least one tendon did not show a similar decrease. One-legged hop-test, Lysholm and IKDC scores significantly increased over time in all groups. An overview of the functional consequences and hamstring tendon regeneration is presented in Table 5 .

Table 5. Functional consequences and hamstring tendon regeneration

\begin{tabular}{|c|c|c|c|c|c|}
\hline & & \multicolumn{2}{|l|}{ Mean (SD) } & \multirow{2}{*}{$\begin{array}{l}\text { Difference } \\
\text { ( } 95 \% \mathrm{Cl} \text { of difference) }\end{array}$} & \multirow[t]{2}{*}{$p$-value } \\
\hline & & To & $\mathrm{T} 2$ & & \\
\hline \multirow[t]{5}{*}{ VAS (at rest) } & $S T+(n=53)$ & $1.1(1.6)$ & $.47(0.9)$ & $.65(.20-1.1)$ & $.005^{*}$ \\
\hline & $G+(n=64)$ & $1.2(1.8)$ & $.52(1.0)$ & $.69(.22-1.2)$ & $.005^{*}$ \\
\hline & $\mathrm{ST}+/ \mathrm{G}+(\mathrm{n}=47)$ & $1.1(1.7)$ & $.52(.95)$ & $.61(.11-1.1)$ & $.017^{*}$ \\
\hline & ST-/G- $(n=9)$ & $.83(.85)$ & $.34(0.45)$ & $.49(-.26-1.2)$ & .17 \\
\hline & control $(n=50)$ & $.71(1.2)$ & $.41(0.75)$ & $.29(-.07-0.66)$ & .11 \\
\hline \multirow{5}{*}{$\begin{array}{l}\text { VAS (during } \\
\text { movement) }\end{array}$} & $S T+(n=53)$ & $2.8(2.5)$ & $.73(.99)$ & $2.1(1.4-2.8)$ & $<.001^{*}$ \\
\hline & $G+(n=64)$ & $2.8(2.6)$ & $.86(1.2)$ & $1.9(1.2-2.6)$ & $<.001^{*}$ \\
\hline & $\mathrm{ST}+/ \mathrm{G}+(\mathrm{n}=47)$ & $2.7(2.5)$ & $.80(1.0)$ & $1.9(1.1-2.6)$ & $<.001^{*}$ \\
\hline & ST-/G- $(n=9)$ & $2.5(2.0)$ & $1.1(1.3)$ & $1.3(-.52-3.2)$ & .14 \\
\hline & control $(n=50)$ & $2.3(2.2)$ & $1.0(1.5)$ & $1.3(.55-2.0)$ & $<.001^{*}$ \\
\hline \multirow[t]{3}{*}{ Tegner } & $S T+(n=53)$ & 8.3 (1.4)\# & $7.1(1.9)$ & $1.2(.68-1.6)$ & $<.001^{*}$ \\
\hline & $G+(n=64)$ & 8.3 (1.4)\# & $6.8(1.9)$ & $1.5(1.0-1.9)$ & $<.001^{*}$ \\
\hline & $\mathrm{ST}+/ \mathrm{G}+(\mathrm{n}=47)$ & 8.3 (1.4)\# & $7.1(1.9)$ & $1.1(.66-1.6)$ & $<.001^{*}$ \\
\hline
\end{tabular}


Table 5. Functional consequences and hamstring tendon regeneration (continued)

\begin{tabular}{|c|c|c|c|c|c|}
\hline & & \multicolumn{2}{|l|}{ Mean (SD) } & \multirow{2}{*}{$\begin{array}{l}\text { Difference } \\
\text { (95\% Cl of difference) }\end{array}$} & \multirow[t]{2}{*}{$p$-value } \\
\hline & & To & $\mathrm{T} 2$ & & \\
\hline & ST-/G- $(n=9)$ & $7.8(1.4) \#$ & $6.4(1.5)$ & $1.3(-.10-32.8)$ & .65 \\
\hline & control $(n=50)$ & 7.5 (1.6)\# & $5.5(2.0)$ & $2.0(1.4-2.6)$ & $<.001^{*}$ \\
\hline \multirow[t]{5}{*}{ Lysholm } & $S T+(n=53)$ & $77.2(13.1)$ & $93.0(7.2)$ & $15.8(12.5-19.2)$ & $<.001^{*}$ \\
\hline & $G+(n=64)$ & $75.4(16.0)$ & $92.8(7.3)$ & $17.4(13.5-21.2)$ & $<.001^{*}$ \\
\hline & $\mathrm{ST}+/ \mathrm{G}+(\mathrm{n}=47)$ & $76.9(13.7)$ & $92.8(7.4)$ & $15.9(12.1-19.6)$ & $<.001^{*}$ \\
\hline & ST-/G- $(n=9)$ & $64.6(11.7)$ & $87.8(15.8)$ & $23.2(9.1-37.3)$ & $.005^{*}$ \\
\hline & control $(n=50)$ & $74.6(16.8)$ & $91.6(12.3)$ & $17.0(11.6-22.4)$ & $<.001^{*}$ \\
\hline \multirow[t]{5}{*}{ IKDC } & $S T+(n=53)$ & $54.4(14.9)$ & $87.6(10.4)$ & $33.2(28.9-37.5)$ & $<.001^{*}$ \\
\hline & $G+(n=64)$ & $52.9(16.3)$ & $87.8(11.2)$ & $34.9(30.4-39.3)$ & $<.001^{*}$ \\
\hline & $S T+/ G+(n=47)$ & $54.2(14.9)$ & $87.9(10.3)$ & $33.7(29.0-38.4)$ & $<.001^{*}$ \\
\hline & ST-/G- $(n=9)$ & $50.8(11.3)$ & $85.2(16.0)$ & $35.4(18.7-50.0)$ & $.001^{*}$ \\
\hline & control $(n=50)$ & $59.2(19.0)$ & $84.3(14.7)$ & $25.1(10.2-30.9)$ & $<.001^{*}$ \\
\hline \multirow{5}{*}{$\begin{array}{l}\text { One-leg hop-test } \\
(\mathrm{cm})\end{array}$} & $S T+(n=53)$ & $53.2(38.7)$ & $110.8(29.4)$ & $57.6(46.8-68.3)$ & $<.001^{*}$ \\
\hline & $G+(n=64)$ & $52.5(38.9)$ & $113.1(30.9)$ & $60.5(50.7-70.4)$ & $<.001^{*}$ \\
\hline & $S T+/ G+(n=47)$ & $54.1(38.6)$ & $111.2(30.1)$ & $57.1(46.2-68.1)$ & $<.001^{*}$ \\
\hline & ST-/G- $(n=9)$ & $37.4(40.2)$ & $94.6(26.6)$ & $57.1(31.1-83.1)$ & $.001^{*}$ \\
\hline & control $(n=50)$ & $57.9(37.9)$ & $101.3(36.5)$ & $43.3(33.4-53.3)$ & $<.001^{*}$ \\
\hline \multirow{5}{*}{$\begin{array}{l}\text { Circumference } \\
\text { upper leg }(\mathrm{cm})\end{array}$} & $S T+(n=53)$ & $45.6(4.8)$ & $45.4(3.7)$ & $.19(-.83-1.2)$ & .71 \\
\hline & $G+(n=64)$ & $47.3(12.8)$ & $45.7(3.6)$ & $1.6(-1.5-4.8)$ & .31 \\
\hline & $\mathrm{ST}+/ \mathrm{G}+(\mathrm{n}=47)$ & $45.8(5.0)$ & $45.6(3.8)$ & $.23(-.90-1.4)$ & .68 \\
\hline & ST-/G- $(n=9)$ & $47.1(4.7)$ & $45.5(4.5)$ & $1.6(.46-2.8)$ & $.01^{*}$ \\
\hline & control $(n=50)$ & $46.6(5.5)$ & $46.3(4.4)$ & $.31(-.62-1.3)$ & .50 \\
\hline
\end{tabular}

VAS (visual analogue score), ST (semitendinosus), G (gracilis), + indicates regeneration, - indicates no regeneration, ${ }^{*}$ pre-trauma Tegner, ${ }^{*}$ p-value $<.05$

\section{Inter correlation coefficient}

The two observers agreed on presence of complete regeneration and absence of regeneration in every case. Assessment of incomplete regeneration was concordant in $95 \%$ of the cases.

The ICC of CSA in the regenerated semitendinosus tendons ranged from 0.97 to 0.99 and for regenerated gracilis tendons from 0.92 to 0.96 .

The ICC of CSA in regenerated semitendinosus tendons ranged from 0.97 to 0.99 and for regenerated gracilis tendons from 0.93 to 0.97 .

\section{Discussion}

Hamstring tendon regeneration occurs in at least $70 \%$ of the patients. ${ }^{37}$ The results of this prospective observational follow-up study show that hamstring tendon regeneration occurs significantly less frequent in patients who smoke. Furthermore,

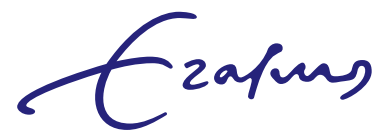


semitendionsus tendons are less likely to regenerate in older patients. Patients without tendon regeneration did not report improved physical activity and demonstrate a significant decrease of their upper leg circumference.

The current study describes that semitendinosus tendons regenerate more often compared to gracilis tendons. This finding is in line with previous studies 38-40 To explain the difference in regenerative capacity, we developed the following hypothesis. It is thought that hamstring tendon regeneration behind the deep layer of the thigh fascia. Regarding this fascia, the gracilis tissue plane is covered and protected to a lesser extent compared to the semitendinosus tendon. This anatomical difference may explain inferior gracilis tendon regeneration rates compared with those of the semitendinosus tendons.

Although previous literature has described several determinants for tendon healing, potential predictive factors for hamstring tendon regeneration have not been investigated before. Therefore, this is the first study that evaluated potential predictive factors for hamstring tendon regeneration, based on known factors for tendon healing. For regeneration of the semitendinosus tendon, we identified age and smoking as predictive factors. Age-related changes in tendons include loss of cellularity, loss of vascularity and fatty infiltration. ${ }^{17}$ Mainly the latter two are thought to be responsible for less regenerative capacity in tendons. The exact mechanism of smoking on hamstring tendon regeneration remains unclear. It could be that nicotin, as a known major vasoconstrictor, affects tendons' regeneration chances by decreasing blood supply to former harvest sites. ${ }^{25}$ On the other hand, nicotine use could also be a marker for unhealthy life styles. However, based on these results it remains hard to predict an individuals' capacity of hamstring tendon regeneration after harvest procedures. As in common orthopaedic pathologies, we therefore suggest a model of intrinsic and extrinsic factors that produce an indication of susceptibility for regenerating processes. However, identifying the cause and genetic linkage of orthopaedic phenotypes has proven to be complex and requires further investigation. So, the current study points out that regeneration of the semitendinosus is related with individuals' age and smoking habits, but it may be that genetic factors also contribute to an individual's regeneration capacity.

This is the first study that examined functional consequences of hamstring tendon regeneration in five different subgroups: regeneration of one tendon only, regeneration of both tendons, no regeneration of both tendons and a non-operatively treated group (control). Although the primary function of the hamstring muscles is to flex the knee or to decelerate extension of the knee, the hamstring muscles also control anterior translation of the tibia, sharing the stress with the ACL. However, we found that all patients experience more knee stability at two years follow-up, regardless regeneration status of the hamstring tendons. Secondly, several studies before used the one-leg hop-test for distance to examine strength

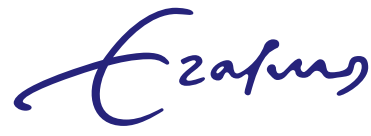


hand confidence in the tested leg. ${ }^{34,35}$ The current study describes that all groups showed a significant increase in the one-leg hop-test results, suggesting that the number of regenerated tendons does not affect clinical performance. An increase of one-leg hop-test results has been previously reported. ${ }^{2}$ However, this study did not differentiate between patients with and without regenerated tendons. In addition, Choi et al. reported no statistically significant difference between the number of regenerated tendons and one-leg hop-test results. ${ }^{11}$ This is in line with the findings of the current study.

Furthermore, we found that the circumference of the operated upper leg is significantly decreased in patients without regeneration, compared to patients that showed regeneration of one or more hamstring tendons. Although this could not be confirmed with measurements in the current study, a previous study reported that the majority of the upper leg atrophy involves the semitendinosus and gracilis muscles. ${ }^{36}$

A previous study investigated the relationship between tendon regeneration, flexor strength and functional tests described in a study of forty-five patients at a minimum follow-up of two years that individual tendon regeneration was associated with less knee flexion deficits at positions of 70 degrees and improved performances on the carioca test. ${ }^{11}$ Taken this together, the results may suggest that no regeneration results in knee flexion deficits because of muscle atrophy of the harvested tendons.

The current study confirms previous studies' findings of a significant atrophy of the harvested tendons. ${ }^{8}, 44$ These studies showed a compensatory hypertrophy of the biceps femoris. However, this could not adequately compensate the loss of muscle volume measured in the harvested medial hamstrings. ${ }^{9,} 36$ Unfortunately, most of these studies compared clinical outcomes postoperatively regardless of an individual's regeneration status. In addition, only relatively short-term follow-up studies are available. So, although there are some strong indications to the clinical relevance of hamstring tendon regeneration, it remains to be seen if different degrees of muscle atrophy and tendon regeneration will have any clinically relevant impact on patient at longer-term follow-ups.

The strengths of the current study are its prospective design, availability of baseline and follow-up MR images, extensive physical examination and questionnaires at baseline and follow-ups. Due to these strengths, we were able to identify predictive factors and clinical consequences of hamstring tendon regeneration in different subgroups.

This study has also some limitations. The used parameters to evaluate clinical consequences of hamstring tendon regeneration may be debatable, as they may be not specific for hamstring tendon regeneration. However, currently there is no specific test to evaluate the function of the semitendinosus and gracilis muscles.

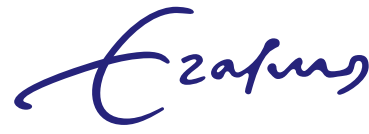


Although there are no functional consequences, determining muscle function using Biodex measurements may be useful. Another limitation of our study is that patients showing regeneration of both the semitendinosus and gracilis tendons were also included in analysis for semitendinosus and gracilis regeneration separately. Therefore, those three groups have a certain overlap.

In conclusion, the current study reported that semitendinosus and gracilis tendons regenerated in $67.1 \%$ and $81.0 \%$ respectively. Furthermore this study points out that regeneration of the semitendinosus is related with individuals' age and smoking habits. Besides, regeneration of both hamstring tendons is negatively related smoking habits. However, absence of regenerated tendons does not seem to cause a loss of function.

\section{REFERENCES}

1. Ahlden M, Samuelsson K, Sernert N, Forssblad M, Karlsson J, Kartus J. The Swedish National Anterior Cruciate Ligament Register: a report on baseline variables and outcomes of surgery for almost 18,000 patients. Am J Sports Med. 2012;40(10):2230-2235.

2. Ahlen $M$, Liden $M$, Bovaller A, Sernert N, Kartus J. Bilateral magnetic resonance imaging and functional assessment of the semitendinosus and gracilis tendons a minimum of 6 years after ipsilateral harvest for anterior cruciate ligament reconstruction. Am J Sports Med. 2012;40(8):1735-1741.

3. Bedi A, Fox AJ, Harris PE, et al. Diabetes mellitus impairs tendon-bone healing after rotator cuff repair. J Shoulder Elbow Surg. 2010;19(7):978-988.

4. Berendsen AD, Smit TH, Walboomers XF, Everts V, Jansen JA, Bronckers AL. Three-dimensional loading model for periodontal ligament regeneration in vitro. Tissue Eng Part $C$ Methods. 2009;15(4):561-570.

5. Bray RC, Leonard CA, Salo PT. Vascular physiology and long-term healing of partial ligament tears. J Orthop Res. 2002;20(5):984-989.

6. Briggs KK, Kocher MS, Rodkey WG, Steadman JR. Reliability, validity, and responsiveness of the Lysholm knee score and Tegner activity scale for patients with meniscal injury of the knee. J Bone Joint Surg Am. 2006;88(4):698-705.

7. Briggs KK, Lysholm J, Tegner Y, Rodkey WG, Kocher MS, Steadman JR. The reliability, validity, and responsiveness of the Lysholm score and Tegner activity scale for anterior cruciate ligament injuries of the knee: 25 years later. Am J Sports Med. 2009;37(5): 890-897.

8. Burks RT, Crim J, Fink BP, Boylan DN, Greis PE. The effects of semitendinosus and gracilis harvest in anterior cruciate ligament reconstruction. Arthroscopy. 2005;21(10):11771185.

9. Carofino B, Fulkerson J. Medial hamstring tendon regeneration following harvest for anterior cruciate ligament reconstruction: fact, myth, and clinical implication. Arthroscopy. 2005;21(10):1257-1265.

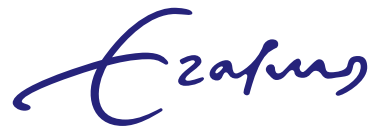


10. Chbinou N, Frenette J. Insulin-dependent diabetes impairs the inflammatory response and delays angiogenesis following Achilles tendon injury. Am J Physiol Regul Integr Comp Physiol. 2004;286(5):R952-957.

11. Choi JY, Ha JK, Kim YW, Shim JC, Yang SJ, Kim JG. Relationships among tendon regeneration on MRI, flexor strength, and functional performance after anterior cruciate ligament reconstruction with hamstring autograft. Am J Sports Med. 2012;40(1):152-162.

12. Cross MJ, Roger G, Kujawa P, Anderson IF. Regeneration of the semitendinosus and gracilis tendons following their transection for repair of the anterior cruciate ligament. Am J Sports Med. 1992;20(2):221-223.

13. Eriksson K, Hamberg P, Jansson E, Larsson H, Shalabi A, Wredmark T. Semitendinosus muscle in anterior cruciate ligament surgery: Morphology and function. Arthroscopy. 2001;17(8):808-817.

14. Ferry ST, Dahners LE, Afshari HM, Weinhold PS. The effects of common anti-inflammatory drugs on the healing rat patellar tendon. Am J Sports Med. 2007;35(8):1326-1333.

15. Granan LP, Forssblad M, Lind M, Engebretsen L. The Scandinavian ACL registries 20042007: baseline epidemiology. Acta Orthop. 2009;80(5):563-567.

16. Hapa O, Cakici H, Gideroglu K, Ozturan K, Kukner A, Bugdayci G. The effect of ethanol intake on tendon healing: a histological and biomechanical study in a rat model. Arch Orthop Trauma Surg. 2009;129(12):1721-1726.

17. Hashimoto T, Nobuhara K, Hamada T. Pathologic evidence of degeneration as a primary cause of rotator cuff tear. Clin Orthop Relat Res. 2003(415):111-120.

18. Irrgang JJ, Anderson AF, Boland AL, et al. Development and validation of the international knee documentation committee subjective knee form. Am J Sports Med. 2001; 29(5):600-613.

19. Janssen RP, van der Velden MJ, Pasmans HL, Sala HA. Regeneration of hamstring tendons after anterior cruciate ligament reconstruction. Knee Surg Sports Traumatol Arthrosc. 2013;21(4):898-905.

20. Jensen MP, Miller L, Fisher LD. Assessment of pain during medical procedures: a comparison of three scales. Clin J Pain. 1998;14(4):343-349.

21. Kocher MS, Steadman JR, Briggs KK, Sterett WI, Hawkins RJ. Reliability, validity, and responsiveness of the Lysholm knee scale for various chondral disorders of the knee. $J$ Bone Joint Surg Am. 2004;86-a(6):1139-1145.

22. Magnussen RA, Granan LP, Dunn WR, et al. Cross-cultural comparison of patients undergoing ACL reconstruction in the United States and Norway. Knee Surg Sports Traumatol Arthrosc. 2010;18(1):98-105.

23. Mallon WJ, Misamore G, Snead DS, Denton P. The impact of preoperative smoking habits on the results of rotator cuff repair. J Shoulder Elbow Surg. 2004;13(2):129-132.

24. Meuffels DE, Poldervaart MT, Diercks RL, et al. Guideline on anterior cruciate ligament injury. Acta Orthop. 2012;83(4):379-386.

25. Mosely LH, Finseth F. Cigarette smoking: impairment of digital blood flow and wound healing in the hand. Hand. 1977;9(2):97-101.

26. Moses B, Orchard J, Orchard J. Systematic review: Annual incidence of ACL injury and surgery in various populations. Res Sports Med. 2012;20(3-4):157-179.

27. Nakamae A, Deie $M$, Yasumoto $M$, et al. Three-dimensional computed tomography imaging evidence of regeneration of the semitendinosus tendon harvested for anterior

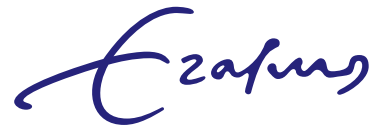


cruciate ligament reconstruction: a comparison with hamstring muscle strength. J Comput Assist Tomogr. 2005;29(2):241-245.

28. Nakamae A, Ochi M, Deie M, Adachi N. Unsuccessful regeneration of the semitendinosus tendon harvested for anterior cruciate ligament reconstruction: report of two cases. Orthop Traumatol Surg Res. 2012;98(8):932-935.

29. Nho SJ, Yadav H, Shindle MK, Macgillivray JD. Rotator cuff degeneration: etiology and pathogenesis. Am J Sports Med. 2008;36(5):987-993.

30. Nishino A, Sanada A, Kanehisa H, Fukubayashi T. Knee-flexion torque and morphology of the semitendinosus after ACL reconstruction. Med Sci Sports Exerc. 2006;38(11):18951900.

31. Nordenvall R, Bahmanyar S, Adami J, Stenros C, Wredmark T, Fellander-Tsai L. A population-based nationwide study of cruciate ligament injury in Sweden, 2001-2009: incidence, treatment, and sex differences. Am J Sports Med. 2012;40(8):1808-1813.

32. O'Connor JP, Lysz T. Celecoxib, NSAIDs and the skeleton. Drugs Today (Barc). 2008;44(9): 693-709.

33. Provenzano PP, Hayashi K, Kunz DN, Markel MD, Vanderby R, Jr. Healing of subfailure ligament injury: comparison between immature and mature ligaments in a rat model. $J$ Orthop Res. 2002;20(5):975-983.

34. Sachs RA, Daniel DM, Stone ML, Garfein RF. Patellofemoral problems after anterior cruciate ligament reconstruction. Am J Sports Med. 1989;17(6):760-765.

35. Seto JL, Orofino AS, Morrissey MC, Medeiros JM, Mason WJ. Assessment of quadriceps/ hamstring strength, knee ligament stability, functional and sports activity levels five years after anterior cruciate ligament reconstruction. Am J Sports Med. 1988;16(2): 170-180.

36. Snow BJ, Wilcox JJ, Burks RT, Greis PE. Evaluation of muscle size and fatty infiltration with MRI nine to eleven years following hamstring harvest for ACL reconstruction. $J$ Bone Joint Surg Am. 2012;94(14):1274-1282.

37. Suijkerbuijk MA, Reijman M, Lodewijks SJ, Punt J, Meuffels DE. Hamstring Tendon Regeneration After Harvesting: A Systematic Review. Am J Sports Med. 2014.

38. Suijkerbuijk MA, Reijman M, Lodewijks SJ, Punt J, Meuffels DE. Hamstring Tendon Regeneration After Harvesting: A Systematic Review. Am J Sports Med. 2015;43(10):25912598.

39. Tadokoro K, Matsui N, Yagi M, Kuroda R, Kurosaka M, Yoshiya S. Evaluation of hamstring strength and tendon regrowth after harvesting for anterior cruciate ligament reconstruction. Am J Sports Med. 2004;32(7):1644-1650.

40. Takeda Y, Kashiwaguchi S, Matsuura T, Higashida T, Minato A. Hamstring muscle function after tendon harvest for anterior cruciate ligament reconstruction: evaluation with T2 relaxation time of magnetic resonance imaging. Am J Sports Med. 2006;34(2): 281-288.

41. van Meer BL, Meuffels DE, Vissers MM, et al. Knee injury and Osteoarthritis Outcome Score or International Knee Documentation Committee Subjective Knee Form: which questionnaire is most useful to monitor patients with an anterior cruciate ligament rupture in the short term? Arthroscopy. 2013;29(4):701-715.

42. Wang JH. Mechanobiology of tendon. J Biomech. 2006;39(9):1563-1582. 
43. West RV, Harner CD. Graft selection in anterior cruciate ligament reconstruction. J Am Acad Orthop Surg. 2005;13(3):197-207.

44. Williams GN, Snyder-Mackler L, Barrance PJ, Axe MJ, Buchanan TS. Muscle and tendon morphology after reconstruction of the anterior cruciate ligament with autologous semitendinosus-gracilis graft. J Bone Joint Surg Am. 2004;86-a(9):1936-1946.

45. Woo SL, Gelberman RH, Cobb NG, Amiel D, Lothringer K, Akeson WH. The importance of controlled passive mobilization on flexor tendon healing. A biomechanical study. Acta Orthop Scand. 1981;52(6):615-622. 